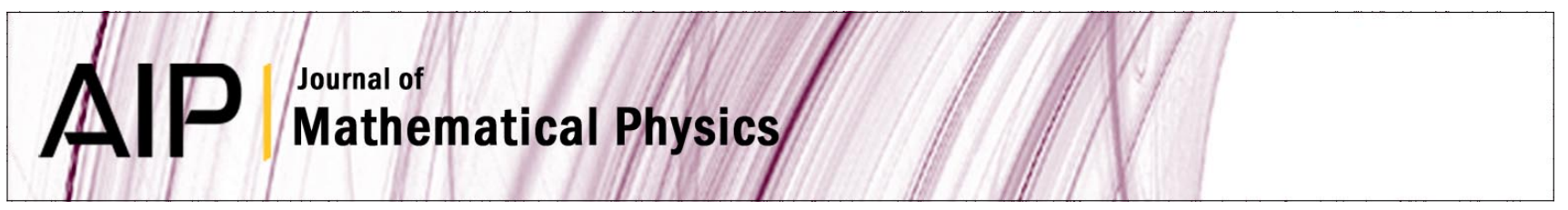

\title{
Collineations of a symmetric 2-covariant tensor: Ricci collineations
}

Josep Llosa

Citation: J. Math. Phys. 54, 072501 (2013); doi: 10.1063/1.4813475

View online: http://dx.doi.org/10.1063/1.4813475

View Table of Contents: http://jmp.aip.org/resource/1/JMAPAQ/v54/i7

Published by the AIP Publishing LLC.

\section{Additional information on J. Math. Phys.}

Journal Homepage: http://jmp.aip.org/

Journal Information: http://jmp.aip.org/about/about_the_journal

Top downloads: http://jmp.aip.org/features/most_downloaded

Information for Authors: http://jmp.aip.org/authors

\section{ADVERTISEMENT}

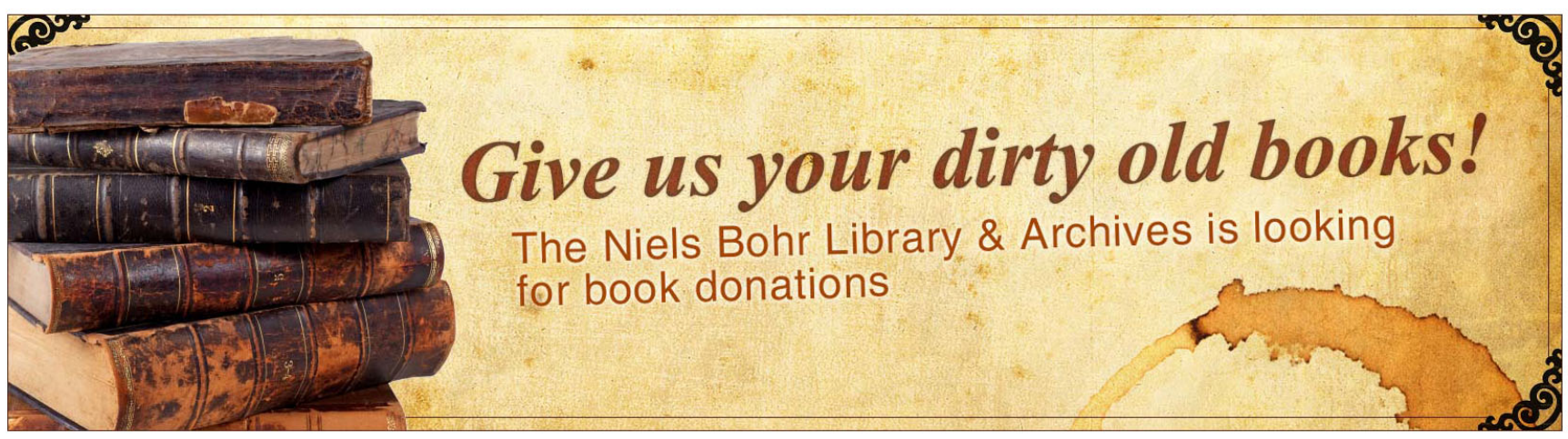




\title{
Collineations of a symmetric 2-covariant tensor: Ricci collineations
}

\author{
Josep Llosa ${ }^{a}$ \\ Departament de Física Fonamental and Institut de Ciències del Cosmos (ICCUB), \\ Universitat de Barcelona, Martí i Franqués 1, E-08028 Barcelona, Spain
}

(Received 25 April 2013; accepted 25 June 2013; published online 17 July 2013)

\begin{abstract}
The infinitesimal transformations that leave invariant a two-covariant symmetric tensor are studied. The interest of these symmetry transformations lays in the fact that this class of tensors includes the energy-momentum and Ricci tensors. We find that in most cases the class of infinitesimal generators of these transformations is a finite dimensional Lie algebra, but in some cases exhibiting a higher degree of degeneracy, this class is infinite dimensional and may fail to be a Lie algebra. As an application, we study the Ricci collineations of a type B warped spacetime. ( 2013 AIP Publishing LLC. [http://dx.doi.org/10.1063/1.4813475]
\end{abstract}

\section{INTRODUCTION}

The interest in the study of symmetries in General Relativity is long-standing. Some of them, namely isometries and affine transformations and their infinitesimal counterparts, Killing vector fields and affine vector fields, are well understood since long ago. ${ }^{1-4}$

In the past 20 years, there has been an steady interest in curvature collineations, Ricci collineations and even matter (Einstein) collineations, ${ }^{5-8}$ to quote a few. Their infinitesimal counterparts, namely collineation fields, are characterized by the vanishing of the Lie derivative of the curvature tensor (resp., the Ricci or the energy-momentum tensor). Collineation fields are thus an extension of the aforementioned Killing fields and affine fields in that every Killing vector field is an affine vector filed which in turn is a curvature collineation field and also a Ricci and a matter collineation field. However, it is well known that collineation fields present new features. Indeed, contrarily to the case of Killing and affine fields, the class $\mathcal{C}$ of curvature (resp., Ricci and matter) collineation fields is a real vector space which may be infinite dimensional; this is due to the dependence on arbitrary functions, which also results in the fact that a collineation field needs not to be smooth and, as a consequence, $\mathcal{C}$ might not be a Lie algebra. ${ }^{7}$

We shall here concentrate in infinitesimal Ricci collineations, but our results are also relevant in the study of curvature collineations because any of them is necessarily a Ricci collineation too. ${ }^{9}$ Contrarily to what is done in most recent literature on the subject, our approach does not use the spacetime metric from which the Ricci tensor is derived; we rather study collineations of a general symmetric 2-covariant tensor because, in our view, paying attention to the metric is rather hindering than helpful.

Properly the results derived here do not apply to the energy-momentum tensor because, from a physical viewpoint, it is rather a $(1,1)$-tensor. Indeed, it is the 4-current density-hence, contravariant-of 4-momentum, which is a covariant magnitude.

Given a 4-manifold $\mathcal{M}$ and a smooth field of symmetric 2-covariant tensors $T$, we shall concentrate on finding the class $\mathcal{C}_{T}$ of vector fields $\mathbf{X}$ such that $\mathcal{L}_{\mathbf{X}} T=0$ and try to find out whether the number of dimensions of $\mathcal{C}_{T}$ is finite, whether $\mathbf{X}$ is smooth and whether $\mathcal{C}_{T}$ is a Lie algebra.

The answer to these questions depend, but not exclusively, on the rank of $T$. Particularly, if the rank is $4, T$ itself can be taken as a non-degenerate metric tensor, the collineation equation is actually

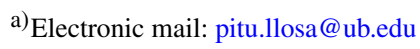


a Killing equation and, as it is well known, ${ }^{1} \mathcal{C}_{T}$ is a subalgebra of the Lie algebra of smooth vector fields, $\mathscr{X}(\mathcal{M})$, and $\operatorname{dim} \mathcal{C}_{T} \leq 10$.

For rank $T<4$, we come across an assorted casuistry which depends not only on the rank of $T$ but also on the derivatives of $T$. We aim to set up a classification of the tensor fields $T$ according to its class $\mathcal{C}_{T}$ of collineation fields. The first variable to consider is the rank and different methods and techniques are suitable for different ranks, e.g., for rank three tensors the method is more similar to that used in studying the Killing fields whereas techniques imported from symplectic mechanics are best suited for rank one tensors. Although it could seem whimsical, the order in which the different ranks are presented here is dictated by their progressive degree of difficulty.

The classification we obtain only holds in a local sense and is rather simple if only the generic, i.e., less degenerate, cases are considered. However as the "degree of degeneracy" (in a sense that will be understood along the way) increases, an intricated mess of cases and subcases arises. We close the paper with an application to type B warped spacetimes.

\section{COLLINEATION FIELDS}

Let $T$ be a 2-covariant symmetric smooth tensor field on a 4-manifold $\mathcal{M}$. A $T$-collineation field (shortly, a collineation field) is a vector field $\mathbf{X}$ such that

$$
\mathcal{L}_{\mathrm{X}} T=0 .
$$

Notice that the definition requires that $\mathbf{X}$ is of class $\mathcal{C}^{1}$ at least but in general it does not guarantee that $\mathbf{X}$ is smooth.

Furthermore, as $\left[\mathcal{L}_{\mathbf{X}}, \mathcal{L}_{\mathbf{Y}}\right]=\mathcal{L}_{[\mathbf{X}, \mathbf{Y}]}$, we have that if $\mathbf{X}$ and $\mathbf{Y}$ are collineation fields of class $\mathcal{C}^{2}$ at least, then $[\mathbf{X}, \mathbf{Y}]$ is also a collineation field. ${ }^{7}$

The case $\operatorname{rank} T=4$ having been discussed, and finished off, in the Introduction, we shall assume that $\operatorname{rank} T=m<4$, constant. Therefore, in the neighbourhood of every $p \in \mathcal{M}$, it exists as a base of $\Lambda^{1} \mathcal{M},\left\{\phi^{a}\right\}_{a=1 \ldots 4}$, such that

$$
T=\eta_{\alpha \beta} \phi^{\alpha} \otimes \phi^{\beta}
$$

with $\eta_{\alpha \beta}=\operatorname{diag}(+1 . r .+1,-1 . s .-1), r+s=m$. (As there is no distinguished metric it is pointless to wonder whether this base is orthonormal or not.) Greek indices run from 1 to $m$, upper case indices $A, B, \ldots$ run from $m+1$ to 4 , latin indices $a, b, \ldots$ run from 1 to 4 and the summation convention is always understood unless the contrary is explicitly stated. The 1 -forms $\phi^{\alpha}$ being independent, we have that $\phi^{1} \wedge \ldots \wedge \phi^{m} \neq 0$ and the set of 1 -forms $\left\{\phi^{\alpha}\right\}_{\alpha=1 \ldots m}$ is called a T-frame.

Let us now expand the Lie derivatives of any $\phi^{v}$ as $\mathcal{L}_{\mathbf{X}} \phi^{v}=M_{\beta}^{v} \phi^{\beta}+M_{B}^{v} \phi^{B}$. Including this and (2) it easily follows that Eq. (1) is equivalent to

$$
\mathcal{L}_{\mathbf{X}} \phi^{\nu}=M_{\beta}^{v} \phi^{\beta}, \quad \eta_{\alpha \nu} M_{\beta}^{v}=\eta_{\beta \nu} M_{\alpha}^{v},
$$

that is, the matrix $M_{\beta}^{v}$ is an $s o(r, s)$-valued function on $\mathcal{M}$.

Any two $T$-frames, $\left\{\phi^{\alpha}\right\}_{\alpha=1 \ldots m}$ and $\left\{\tilde{\phi}^{\alpha}\right\}_{\alpha=1 \ldots m}$, are connected through an $\eta$-orthogonal transformation:

$$
\tilde{\phi}^{\alpha}:=R_{\beta}^{\alpha} \phi^{\beta}, \quad \text { where } \quad R_{\alpha}^{\mu} R_{\beta}^{\nu} \eta_{\mu \nu}=\eta_{\alpha \beta},
$$

i.e., $R_{\beta}^{\alpha}$ is a field of $O(r, s)$ matrices. For the sake of brevity, we shall refer hereafter to these transformations as T-rotations.

$T$ is said to be holonomous if its associated system, $\mathcal{H}_{T}=\left\{\mathbf{Y} \in \mathscr{X}(\mathcal{M}) \mid T\left(\mathbf{Y},{ }_{-}\right)=0\right\}$, is integrable. Then local charts $\left(y^{a}\right)$ exist such that $\phi^{\alpha}=a_{\beta}^{\alpha}\left(y^{b}\right) d y^{\beta}$ (see Ref. 10, Lemma V.4.10) and

$$
T=T_{\alpha \beta}\left(y^{b}\right) d y^{\alpha} \otimes d y^{\beta}, \quad \operatorname{det} T_{\alpha \beta} \neq 0, \quad \mathbf{X}=X^{\alpha} \partial_{\alpha}+X^{A} \partial_{A},
$$

where $T_{\alpha \beta}=\eta_{\mu \nu} a_{\alpha}^{\mu} a_{\beta}^{v}$ and $\partial_{a}:=\frac{\partial}{\partial y^{a}}$. 


\section{COLLINEATIONS OF A RANK 3 TENSOR}

If rank $T=3$, it is obvious that $T$ is holonomous and local charts exist such that the expressions (5) hold. We write the collineation field as $\mathbf{X}=\mathbf{Z}+f \partial_{4}$, where $\mathbf{Z}=Z^{\alpha} \partial_{\alpha}$ is tangential to the submanifolds $y^{4}=$ constant and $f$ is a function.

As $T_{4 a}=0$, Eq. (1) amounts to

$$
\mathcal{L}_{\mathbf{Z}} T_{a b}+f \partial_{4} T_{a b}=0
$$

whose components $4 a$ and $\alpha \beta$ are

$$
\partial_{4} Z^{\alpha}=0 \quad \text { and } \quad \nabla_{(\alpha} Z_{\beta)}+f K_{\alpha \beta}=0,
$$

respectively, where $K_{\alpha \beta}:=\frac{1}{2} \partial_{4} T_{\alpha \beta}$ and $\nabla$ is the Levi-Civita connection for the non-degenerate metric $T_{\alpha \beta}$ on the hypersurfaces $y^{4}=$ constant. The second of these equations looks like a nonhomogeneous Killing equation (parametrized with $y^{4}$ ) and the question is: does it admit solutions $Z^{\alpha}$ that do not depend on $y^{4}$ for some appropriate $f$ ?

If $K_{\alpha \beta}=0$, the answer is obviously yes, because it reduces to a Killing equation in 3 dimensions. The collineation field is then $\mathbf{X}=\mathbf{Z}+f \partial_{4}$, where $f$ is arbitrary and $\mathbf{Z}$ is a Killing vector for the non-degenerate metric $T_{\alpha \beta}$ in each 3-submanifold $y^{4}$ constant.

If $K_{\alpha \beta} \neq 0$, things are not so simple. The second of Eq. (7) implies that

$$
\nabla_{\alpha} Z_{\beta}=\Omega_{\alpha \beta}-f K_{\alpha \beta}, \quad \Omega_{\alpha \beta}+\Omega_{\beta \alpha}=0 .
$$

Their integrability conditions imply new equations on $\Omega_{\alpha \beta}$ and $f$. These can be derived by means of the Lie derivative of a connection (see Ref. 2, Sec. I.4)

$$
\mathcal{L}_{\mathbf{Z}} \nabla_{\mu} T_{\kappa \lambda}-\nabla_{\mu} \mathcal{L}_{\mathbf{Z}} T_{\kappa \lambda}=-\left(\mathcal{L}_{\mathbf{Z}} \Gamma_{\mu \kappa}^{\alpha}\right) T_{\alpha \lambda}-\left(\mathcal{L}_{\mathbf{Z}} \Gamma_{\mu \lambda}^{\alpha}\right) T_{\kappa \alpha},
$$

which after a little algebra yields

$$
\nabla_{\mu} \Omega_{\kappa \lambda}=Z^{\rho} R_{\rho \mu \kappa \lambda}+\nabla_{\lambda}\left(f K_{\mu \kappa}\right)-\nabla_{\kappa}\left(f K_{\mu \lambda}\right) .
$$

On their turn, these equations on $\Omega_{\kappa}$ produce new integrability conditions that involve $\mathcal{L}_{\mathrm{Z}} R_{v \mu \lambda \kappa}$ and, as the latter has the kind of symmetries of a Riemann tensor in three effective dimensions, they amount to one of its traces, that is,

$$
\mathcal{L}_{\mathbf{Z}} R_{\nu \lambda}=2 f\left(K^{\mu \kappa} R_{\nu \mu \lambda \kappa}-R_{(\nu}^{\alpha} K_{\lambda) \alpha}\right)+\nabla^{\alpha} \nabla_{\alpha}\left(f K_{\nu \lambda}\right)+\nabla_{\nu} \nabla_{\lambda}\left(f K_{\mu}^{\mu}\right)-2 \nabla_{(\nu} \nabla_{\alpha}\left(f K_{\lambda)}^{\alpha}\right) .
$$

Then, similarly as in the theory of Killing vectors (see Ref. 1, Chap. 8), using a relation analogous to (9) for general tensors—-see Ref. 2, Eq. (I.4.9)—we obtain the hierarchy of integrability conditions

$$
\mathcal{L}_{\mathbf{Z}} \nabla_{\alpha_{1}} \ldots \nabla_{\alpha_{n}} R_{\nu \lambda}=H_{\alpha_{1} \ldots \alpha_{n} \nu \lambda}\left(f, f_{\mid \beta} \ldots\right),
$$

where $f_{\mid \alpha \ldots \beta}:=\nabla_{\beta} \ldots \nabla_{\alpha} f$.

So far we have analysed the integrability conditions derived from the commutation of $\nabla_{\mu}$ and $\nabla_{v}$. Let us now apply the commutation relations for $\nabla_{v}$ and $\partial_{4}$ to Eq. (7); we obtain that

$$
\begin{aligned}
& \partial_{4} \Omega_{\mu \nu}=2 \Omega_{\lambda[v} K_{\mu]}^{\lambda}+2 Z^{\lambda} \nabla_{[\mu} K_{v] \lambda}, \\
& \mathcal{L}_{\mathbf{Z}} K_{\beta}^{\mu}+\dot{f} K_{\beta}^{\mu}+f \dot{K}_{\beta}^{\mu}=0 .
\end{aligned}
$$

Unless $\dot{K}_{\beta}^{\mu} \propto K_{\beta}^{\mu}$, the latter permits to derive $f=f\left(Z^{\lambda}, \Omega_{\mu \nu}\right)$. Indeed, if $\dot{K}_{\beta}^{\mu}$ is not proportional to $K_{\beta}^{\mu}$, it exists $M_{\mu}^{\alpha}$ such that $M_{\mu}^{\alpha} K_{\alpha}^{\mu}=0$ and $M_{\mu}^{\alpha} \dot{K}_{\alpha}^{\mu}=1$; therefore, $f=-M_{\mu}^{\alpha} \mathcal{L}_{\mathbf{Z}} K_{\alpha}^{\mu}$. (Were there more than one independent matrix $M_{\beta}^{\alpha}$ fulfilling the above trace equalities, it would result in constraints connecting $Z^{\alpha}$ and $\Omega_{\mu \beta}$.)

Substituting then this $f$ in Eqs. (7), (10), and (12), we obtain a closed partial differential system on $Z^{\alpha}$ and $\Omega_{\mu \nu}$. If it is integrable, each solution is parametrized by six real numbers, namely $Z^{\alpha}(0)$ and $\Omega_{\mu \nu}(0)$. The above mentioned hierarchy of integrability conditions then act as constraints on these parameters and the number of dimensions of the collineation algebra $\mathcal{C}_{T}$ is at most six. 
If, on the contrary, $\dot{K}_{\beta}^{\mu}=b K_{\beta}^{\mu}$, then Eq. (13) implies that

$$
\mathcal{L}_{\mathbf{Z}} K_{\beta}^{\mu}+(\dot{f}+b f) K_{\beta}^{\mu}=0, \quad \text { for some } b,
$$

which allows to derive $\dot{f}$ as a linear function of $Z^{\alpha}, \Omega_{\mu \nu}$, and $f$.

Now, applying $\partial_{4}$ to both sides of Eq. (10) and including the commutation relations for $\nabla_{\mu}$ and $\partial_{4}$, after some algebra we arrive at

$$
f_{\mid \alpha}\left(K^{\lambda \alpha} K_{\mu \kappa}-K_{\mu \beta} K_{\kappa}^{\beta} T^{\alpha \lambda}\right)=W_{\mu \kappa}^{\lambda},
$$

where $W_{\mu \kappa}^{\lambda}$ is a linear function of $Z^{\alpha}$ and $\Omega_{\mu \nu}$. In many cases, this permits to obtain $f_{\mid \alpha}$ as a unique linear function of $Z^{\alpha}, \Omega_{\mu \beta}$, and $f$. This happens whenever the linear map $K^{\lambda \alpha} K_{\mu \kappa}-K_{\mu \beta} K_{\kappa}^{\beta} T^{\alpha \lambda}$ is injective and the right-hand side $W_{\mu \kappa}^{\lambda}$ fulfills some compatibility conditions, that amount to some linear constraints on $Z^{\alpha}, \Omega_{\mu \beta}$, and $f$.

This expression for $f_{\mid \alpha}$ as a linear function of $Z^{\alpha}, \Omega_{\mu \beta}$, and $f$, together with (8), (10), (12) and (14), yields a partial differential system on the variables $Z^{\alpha}, \Omega_{\mu \nu}$, and $f$. If it is integrable, each solution is parametrized by the seven real numbers $Z^{\alpha}(0), \Omega_{\mu \nu}(0)$, and $f(0)$, and the collineation algebra $\mathcal{C}_{T}$ has at most seven dimensions.

The highly degenerate cases in which Eq. (15) cannot be solved for $f_{\mid \alpha}$ require further analysis.

\section{COLLINEATIONS OF A RANK 1 TENSOR}

If rank $T=1$, then it exists $\phi \in \Lambda^{1} \mathcal{M}$ such that $T= \pm \phi \otimes \phi$, and the collineation condition (1) is equivalent to $\mathcal{L}_{\mathbf{X}} \phi=0$, which means that, locally, a function $f$ exists such that

$$
\begin{aligned}
& i_{\mathbf{X}} \phi=f, \\
& i_{\mathbf{X}} d \phi=-d f .
\end{aligned}
$$

This is a linear system on $\mathbf{X}$ whose compatibility depends on $f$ and on the class of the differential form $\phi$. We shall need the following corollary of Darboux theorem—see Ref. 10, Theorem VI.4.1.

Theorem 1: Given $\phi \in \Lambda^{1}(M)$, they exist a canonical coordinate system $p_{1}, p_{2}, q^{1}, q^{2}$, and a function $\psi$, such that

$$
\phi=d \psi+e_{1} p_{1} d q^{1}+e_{2} p_{2} d q^{2}
$$

with $e_{1} \geq e_{2} \quad e_{1}, e_{2}=0,1$.

A remark on notation is appropriate: hereon a stroke means partial derivative, so $v_{\mid a}:=\partial_{a} v:=$ $\partial v / \partial x^{a} ;$ particularly in canonical coordinates $\left(q^{i}, p_{j}\right)$,

$$
v_{\mid i}:=\partial_{i} v:=\frac{\partial v}{\partial q^{i}} \quad \text { and } \quad v^{\mid j}:=\partial^{j} v:=\frac{\partial v}{\partial p_{j}},
$$

with $i, j=1,2$. Writing now $\mathbf{X}$ and $d f$ in canonical coordinates,

$$
\mathbf{X}=X^{i} \partial_{i}+X_{i} \partial^{i}, \quad d f=f_{\mid i} d q^{i}+f^{\mid i} d p_{i},
$$

Eq. (16b) amounts to

$$
-e_{i} X_{i}=f_{\mid i}, \quad e_{i} X^{i}=f^{\mid i} .
$$

Then, including this and (17), we obtain that Eq. (16a) amounts to

$$
X^{i} \phi_{i}+X_{i} \phi^{i}=f
$$

with $\phi_{i}:=\psi_{\mid i}+e_{i} p_{i}$ and $\phi^{i}:=\psi^{\mid i}$. According to the values of $e_{1}$ and $e_{2}$, different cases are possible, which we shall analyse separately. 
[1.nd]: $(d \phi)^{2} \neq 0$. Then $\Omega:=d \phi$ is a symplectic form and $e_{1}=e_{2}=1$.

In this case, the class of the differential form $\phi$ is 4 (see Ref. 10, Section VI.1.3) and Darboux theorem states more precisely that canonical local charts exist such that $\psi=0$, that is, $\phi=$ $p_{1} d q^{1}+p_{2} d q^{2}$. Equation (18) then implies that

$$
\mathbf{X}=-\{f,-\}=f^{i} \partial_{i}-f_{i} \partial^{i},
$$

where $\{$,$\} is the Poisson bracket for d \phi$. Including this, Eq. (19) becomes

$$
\sum_{i=1}^{2} p_{i} f^{\mid i}=f,
$$

which, by Euler theorem, means that $f\left(q^{i}, p_{j}\right)$ is an homogeneous function of the first degree in the variables $p_{j}$. The general collineation field is thus $\mathbf{X}=-\{f,-\}$, where $f \in \Lambda^{0} \mathcal{M}$ is a solution of (20).

[1.d]: $(d \phi)^{2}=0$ but $d \phi \wedge \phi \neq 0$ which, including Darboux Theorem, implies that canonical coordinates exist such that $\phi=d q^{2}+p_{1} d q^{1}$, i.e., $e_{2}=0$ and $e_{1}=1$. Combining then Eqs. (19) and (18), we obtain that the general collineation field is

$$
\mathbf{X}=f^{\mid 1} \partial_{1}-f_{\mid 1} \partial^{1}+\left(f-p_{1} f^{\mid 1}\right) \partial_{2}+X_{2} \partial^{2},
$$

where $f\left(p_{1}, q^{1}\right)$ and $X_{2}\left(p_{i}, q^{j}\right)$ are arbitrary functions of their respective variables.

[1.d.h]: $(d \phi)^{2}=d \phi \wedge \phi=0$ but $d \phi \neq 0$. In this case, $\phi$ is integrable and a local chart exists such that $\phi=p_{1} d q^{1}$. Combining then Eqs. (19) and (21), we obtain that

$$
f-p_{1} f^{11}=0 \quad \text { or, } \quad f=p_{1} F\left(q^{1}\right) .
$$

There is no constraint on the components $X^{2}$ and $X_{2}$ and the general collineation field,

$$
\mathbf{X}=F\left(q^{1}\right) \partial_{1}-p_{1} F^{\prime}\left(q^{1}\right) \partial^{1}+X^{2} \partial_{2}+X_{2} \partial^{2},
$$

contains three arbitrary functions, namely $F\left(q^{1}\right), X^{2}\left(p_{i}, q^{j}\right)$, and $X_{2}\left(p_{i}, q^{j}\right)$.

[1.d.0]: $d \phi=0$. Then, there exist locals charts, $\left\{x^{a}\right\}_{a=1 \ldots 4}$, such that, $\phi=d x^{1}$, and the general solution to the collineation equation (1) is

$$
\mathbf{X}=C \frac{\partial}{\partial x^{1}}+\sum_{\nu=2}^{4} X^{\nu} \frac{\partial}{\partial x^{\nu}}
$$

with $X^{\nu}\left(x^{a}\right)$ arbitrary and $C$ constant.

\section{COLLINEATIONS OF A RANK 2 TENSOR}

Now $T=\eta_{\alpha \beta} \phi^{\alpha} \otimes \phi^{\beta}$, with $\eta_{\alpha \beta}=\operatorname{diag}(1, \pm 1)$. In what follows, it will be helpful to consider the 2 -forms $d \phi^{\alpha}$ and the exterior products

$$
\Upsilon^{\alpha}:=d \phi^{\alpha} \wedge \phi^{1} \wedge \phi^{2} \quad \text { and } \quad \Sigma^{\alpha \beta}:=d \phi^{\alpha} \wedge d \phi^{\beta} .
$$

A $T$-rotation (4) is defined by a matrix $R_{\beta}^{\alpha}(\zeta)$ which depends on a function $\zeta$ and we have that

$$
d \tilde{\phi}^{\alpha}=\dot{R}_{\beta}^{\alpha} d \zeta \wedge \phi^{\beta}+R_{\beta}^{\alpha} d \phi^{\beta},
$$

where $\dot{R}_{\beta}^{\alpha}:=\partial_{\zeta} R_{\beta}^{\alpha}$, and

$$
\tilde{\phi}^{1} \wedge \tilde{\phi}^{2}=\operatorname{det}\left(R_{\beta}^{\alpha}\right) \phi^{1} \wedge \phi^{2} .
$$


Now, as $\operatorname{det}\left(R_{\beta}^{\alpha}\right)= \pm 1$, it follows that

$$
\begin{gathered}
\tilde{\Upsilon}^{\alpha}= \pm R_{\beta}^{\alpha} \Upsilon^{\beta}, \\
\tilde{\Sigma}^{\alpha \beta}=R_{\mu}^{\alpha} R_{\nu}^{\beta} \Sigma^{\mu \nu}+\left(R_{\nu}^{\alpha} \dot{R}_{\mu}^{\beta}+R_{\nu}^{\beta} \dot{R}_{\mu}^{\alpha}\right) d \zeta \wedge \phi^{\mu} \wedge d \phi^{v} .
\end{gathered}
$$

Now, let $\Omega \in \Lambda^{4} \mathcal{M}$ be a volume tensor $(\Omega \neq 0)$ and define $l^{\alpha}$ by $\Upsilon^{\alpha}=l^{\alpha} \Omega$. The relation (23) implies that $\tilde{l}^{\alpha}:= \pm R_{\beta}^{\alpha} l^{\beta}$ and, as a consequence,

$$
\eta_{\alpha \beta} l^{\alpha} l^{\beta} \text { is invariant by } T-\text { rotations. }
$$

Therefore, unless $\eta_{\alpha \beta}=\operatorname{diag}(1,-1)$ and $\Upsilon^{1}=\Upsilon^{2}$, we can always perform a $T$-rotation such that one of the exterior products $\Upsilon^{\alpha}$ vanishes (we can label the 1-forms $\phi^{\beta}$ so that this is $\Upsilon^{1}$ ) and $T$ can be classified in one of the following types:

\begin{tabular}{llll}
\hline \hline 2.I & $\Upsilon^{1}=0, \quad \Upsilon^{2} \neq 0$ & $\mathbf{a}$ & $\Sigma^{11} \neq 0$ \\
2.N & $\Upsilon^{1}=\Upsilon^{2} \neq 0$ & $\mathbf{b}$ & $\Sigma^{11}=0$ \\
$\mathbf{2 . H}$ & $\Upsilon^{1}=\Upsilon^{2}=0$ & & \\
\hline \hline
\end{tabular}

[Notice that Type 2.N only occurs if $\eta_{\alpha \beta}$ has no defined sign.]

\section{A. Type 2.I.a}

A little algebra allows to proof the following.

Proposition 1: If $\Sigma^{11} \neq 0, \Upsilon^{1}=0$ and $\Upsilon^{2} \neq 0$, then two differential forms $\phi^{A} \in \Lambda^{1} \mathcal{M}, A=$ 3,4 , exist such that

$$
\begin{aligned}
& d \phi^{1}=\phi^{3} \wedge \phi^{1}+\phi^{4} \wedge \phi^{2} \\
& d \phi^{2}=r d \phi^{1}+\frac{s-r^{2}}{2 l} \phi^{1} \wedge \phi^{2}-2 l \phi^{3} \wedge \phi^{4}
\end{aligned}
$$

where

$$
\Sigma^{12}=r \Sigma^{11}, \quad \Sigma^{22}=s \Sigma^{11} \text { and } \Upsilon^{2}=l \Sigma^{11}
$$

with $l \neq 0$. The differential forms $\phi^{A}$ are uniquely determined and $\left\{\phi^{a}\right\}_{a=1 \ldots 4}$ is the canonical base for the tensor $T$.

As $\operatorname{rank} T=2$, Eq. (3) reads

$$
\mathcal{L}_{\mathbf{X}} \phi^{\alpha}=b D_{\beta}^{\alpha} \phi^{\beta}
$$

$\alpha, \beta=1,2$, where $D_{1}^{1}=D_{2}^{2}=0, D_{2}^{1}=1, D_{1}^{2}=-\sigma, \sigma= \pm 1$ and $b$ is a function. Then it follows that $\mathcal{L}_{\mathbf{X}}\left(\phi^{1} \wedge \phi^{2}\right)=0$ and

$$
\mathcal{L}_{\mathbf{X}} \Upsilon^{1}=b \Upsilon^{2}
$$

Now, as $\Upsilon^{1}=0$ and $\Upsilon^{2} \neq 0$, it follows that $b=0$ which, substituted in (27) yields

$$
\mathcal{L}_{\mathbf{X}} \phi^{\alpha}=0, \alpha=1,2 .
$$

On its turn, this implies that $\mathcal{L}_{\mathbf{X}} d \phi^{\alpha}=0$ which, including Eq. (25), leads to

$$
\mathcal{L}_{\mathbf{X}} \phi^{A}=0, A=3,4 .
$$

Summarizing, if $T$ is type 2.I.a, first we find the canonical base $\left\{\phi^{a}\right\}_{a}=1 \ldots 4$ and its dual base $\left\{\mathbf{Y}_{a}\right\}_{a=1 \ldots 4}$. Then the collineation equations supplemented with their integrability conditions amount 
to $\mathcal{L}_{\mathbf{X}} \phi^{a}=0$ which, writing $\mathbf{X}=X^{a} \mathbf{Y}_{a}$ and $d \phi^{a}=-\frac{1}{2} C_{b c}^{a} \phi^{b} \wedge \phi^{c}$, amounts to

$$
d X^{a}-X^{b} C_{b c}^{a} \phi^{c}=0 .
$$

If this partial differential system is integrable, each solution is parametrized by the values $X_{0}^{b}$ at one point. Therefore, the dimension of the collineation algebra for type 2.I.a tensors is at most 4 .

The integrability conditions of (30) put some further constraints on the parameters $X_{0}^{b}$. These integrability conditions are obtained by taking the exterior derivative and read $\mathcal{L}_{\mathbf{X}} d \phi^{a}=0$ or, in terms of the coefficients $C_{b c}^{a}$,

$$
\mathbf{X} C_{b c}^{a}=0
$$

which eventually implies that

$$
\left.C_{b c \mid e_{1} \ldots e_{n} h}^{a}\right|_{0} X_{0}^{h}=0, \quad n \in \mathbb{N} .
$$

This is an infinite homogeneous linear system on the parameters $X_{0}^{b}$. Provided that its rank is not greater than 4 , the codimension of the collineation algebra for type 2.I.a tensors is precisely this rank, otherwise $T$ admits no collineation fields.

\section{B. Type 2.I.b}

Again, a little algebra allows to proof that

Proposition 2: If $\Upsilon^{2} \neq 0, \Upsilon^{1}=0$ and $\Sigma^{11}=0$, then two differential forms $\phi^{A} \in \Lambda^{1} \mathcal{M}, A=$ 3,4 , exist such that

$$
\begin{aligned}
& d \phi^{2}=\frac{s}{2} \phi^{1} \wedge \phi^{2}+\phi^{3} \wedge \phi^{4}, \\
& d \phi^{1}=r \phi^{1} \wedge \phi^{2}+v_{\alpha} \phi^{\alpha} \wedge \phi^{3} .
\end{aligned}
$$

where $v_{\alpha}$ is $(0,0),(1, v)$ or $(v, 1)$. Besides $\Sigma^{12}=r \Upsilon^{2}$ and $\Sigma^{22}=s \Upsilon^{2}$.

The differential form $\phi^{4}$ is determined up to the gauge transformation, $\phi^{\prime 4}=\phi^{4}+m \phi^{3}$, where $m$ is an arbitrary function.

The collineation equation (3) amounts to Eq. (27), which implies that $\mathcal{L}_{\mathbf{X}} \Upsilon^{1}=b \Upsilon^{2}$ and, as $\Upsilon^{1}=0$ and $\Upsilon^{2} \neq 0$, it follows that $b=0$ and therefore

$$
\mathcal{L}_{\mathbf{X}} \phi^{\alpha}=0, \quad \alpha=1,2 .
$$

Now two cases must be separately considered.

Case 2.I.b.1: If $v_{\alpha} \neq 0$, then either $v_{\alpha}=(1, v)$ or $v_{\alpha}=(v, 1)$, and the integrability conditions for Eq. (34) imply that $\mathcal{L}_{\mathbf{X}} d \phi^{\alpha}=0$, which, with a little algebra, lead to

$$
\mathcal{L}_{\mathbf{X}} \phi^{3}=0, \quad \mathcal{L}_{\mathbf{X}} \phi^{4} \wedge \phi^{3}=0 \quad \text { and } \quad \mathbf{X} v=0 .
$$

Equations (34) and (35) can then be unified as

$$
\mathcal{L}_{\mathbf{X}} \phi^{a}=\delta_{4}^{a} f \phi^{3}, \quad \text { for some function } f,
$$

which is equivalent to

$$
d X^{a}=\left(X^{e} C_{e c}^{a}+f \delta_{4}^{a} \delta_{c}^{3}\right) \phi^{c},
$$

where as before $\mathbf{X}=X^{a} \mathbf{Y}_{a}$ and $C_{b c}^{a}$ are the commutation coefficients in this base. Due to the occurrence of the unknown function $f$, this partial differential system is not in closed form. However, in most generic cases the integrability conditions could help to determine $f$.

The integrability condition for Eq. (36), $a=4$, yields

$$
\begin{aligned}
& \qquad M=f\left(C_{4 \beta}^{4} \phi^{3} \wedge \phi^{\beta}+d \phi^{3}\right)+d f \wedge \phi^{3} \\
& \text { with } \quad M:=-\frac{1}{2} \mathbf{X} C_{c b}^{4} \phi^{c} \wedge \phi^{b} .
\end{aligned}
$$


Now, if $\phi^{3} \wedge d \phi^{3} \neq 0$, we can obtain $f=f\left(X^{c}\right)$, which closes the differential system (36). If it is integrable, then the solution depends on the four real parameters $X_{0}^{a}$, which are subject to the hierarchy of constraints that follow from the full integrability conditions of the system (36), and $\operatorname{dim} \mathcal{C}_{T} \leq 4$.

If, on the contrary, $\phi^{3} \wedge d \phi^{3}=0$, after some elaboration we arrive at

$$
f \phi^{3} \wedge d\left(C_{4 \beta}^{4} \phi^{\beta}\right)=X^{a} F_{a c b} \phi^{3} \wedge \phi^{c} \wedge \phi^{b},
$$

where $F_{a c b}$ are some coefficients that do not depend on $X^{e}$. Then, provided that the left-hand side does not vanish, we can derive $f=f\left(X^{a}\right)$, which closes the partial differential system (37), and therefore $\operatorname{dim} \mathcal{C}_{T} \leq 4$.

We do not analyse here the highly non-generic case that neither Eq. (37) nor Eq. (38) can be solved for $f$, which would require further study.

Case 2.I.b.O: If $v_{\alpha}=0$, then it follows from (33) that

$$
d \phi^{2}=\frac{s}{2} \phi^{1} \wedge \phi^{2}+\phi^{3} \wedge \phi^{4} \quad \text { and } \quad d \phi^{1}=r \phi^{1} \wedge \phi^{2} .
$$

The integrability of the latter implies that $r=0$, i.e., $d \phi^{1}=0$, and locally a function $y$ exists such that $\phi^{1}=d y$. Equation (34) then implies that $\mathbf{X} y=C$, constant, and two cases must be considered depending on whether $s$ does vanish or not.

2.I.b.0.nd If $s \neq 0$, then $d \phi^{2}$ is symplectic and, using canonical coordinates for $\phi^{2}$, we can apply the results in Sec. IV, case 1.nd to obtain that $\mathbf{X}=-\{f,-\}$, where

$$
p_{i} \partial^{i} f=f, \quad\{y, f\}=C .
$$

Let $\mathcal{H}$ be the minimal integrable distribution containing $\mathbf{P}=p_{j} \partial^{j}$ and $\mathbf{Y}=\left\{y,-{ }_{-}\right\}$, then $2 \leq \operatorname{dim} \mathcal{H} \leq 4$ and $\operatorname{dim} \mathcal{H}^{\perp}$ is the number of arbitrary functions on which $f$ depends.

2.I.b.0.d If $s=0$, then $d \phi^{2} \wedge d \phi^{2}=0$ and, as $\Upsilon^{2} \neq 0$, we also have that $d \phi^{2} \wedge \phi^{2} \neq 0$. The results in Sec. IV, case 1.d apply and canonical coordinates can be chosen such that $\phi^{1}=$ $d p_{2}, \phi^{2}=d q^{2}+p_{1} d q^{1}$ and

$$
\mathbf{X}=f^{\mid 1} \partial_{1}-f_{\mid 1} \partial^{1}+\left(f-p_{1} f^{\mid 1}\right) \partial_{2}+C \partial^{2},
$$

where $f=f\left(p_{1}, q^{1}\right)$.

\section{Type 2.N}

This case only happens when $\eta_{\alpha \beta}=\operatorname{diag}(1,-1)$, i.e., $\sigma=-1$, and we shall write

$$
\Sigma^{11}=t \Upsilon^{1}, \quad \Sigma^{12}=r \Upsilon^{1}, \quad \Sigma^{22}=s \Upsilon^{1} .
$$

With a little algebra, it can be easily proven that, as $\Upsilon^{1}=\Upsilon^{2} \neq 0$, two differential forms, $\phi^{3}$ and $\phi^{4}$, exist such that

$$
\left.\begin{array}{l}
d \phi^{1}=\frac{t}{2} \phi^{1} \wedge \phi^{2}+\phi^{3} \wedge \phi^{4} \\
d\left(\phi^{2}-\phi^{1}\right)=(r-t) \phi^{1} \wedge \phi^{2}+P_{\alpha \beta} \phi^{\alpha} \wedge \phi^{\beta+2}
\end{array}\right\}
$$

with $\operatorname{det} P_{\alpha \beta}=r-(s+t) / 2, \alpha, \beta=1,2$, and the differential forms $\phi^{\alpha+2}$ are determined up to the gauge transformation $\phi^{\prime \alpha+2}=L_{\nu}^{\alpha} \phi^{\nu+2}$ where $L_{\nu}^{\alpha}$ is a $S L(2)$-valued function.

The value of $\operatorname{det}\left(P_{\alpha \beta}\right)$ is $T$-frame dependent. Indeed, by a $T$-rotation the volume forms $\Sigma^{\alpha \beta}$ transform according to (24) and, including that

$$
R_{v}^{\alpha}=\left(\begin{array}{cc}
\cosh \zeta & \sinh \zeta \\
\sinh \zeta & \cosh \zeta
\end{array}\right)
$$


and that $\tilde{\Upsilon}^{1}=e^{\zeta} \Upsilon^{1}$, we finally arrive at

$$
e^{3 \zeta}(\tilde{t}+\tilde{s}-2 \tilde{r})=(t+s-2 r)+2\left(P_{24}+P_{14}\right) \zeta_{3}-2\left(P_{23}+P_{13}\right) \zeta_{4},
$$

where $d \zeta=\zeta_{a} \phi^{a}$.

If $\operatorname{det}\left(P_{\alpha \beta}\right) \neq 0$, then the coefficients of $\zeta_{3}$ and $\zeta_{4}$ cannot vanish simultaneously and the latter equation can be used to obtain a $T$-frame in which $\operatorname{det}\left(\tilde{P}_{\alpha \beta}\right)=0$.

Furthermore, if $\operatorname{det}\left(P_{\alpha \beta}\right)=0$, the above mentioned gauge freedom can be used to chose $\phi^{3}$ and $\phi^{4}$ so that $P_{\alpha \beta}=v_{\alpha} \delta_{\beta}^{1}$. These results can be summarized as

Proposition 3: For type 2.N tensors it always exists a T-frame such that

$$
\begin{gathered}
d \phi^{1}=\frac{t}{2} \phi^{1} \wedge \phi^{2}+\phi^{3} \wedge \phi^{4}, \\
d\left(\phi^{2}-\phi^{1}\right)=\frac{s-t}{2} \phi^{1} \wedge \phi^{2}+\left[w\left(\phi^{2}-\phi^{1}\right)+u \phi^{2}\right] \wedge \phi^{3}
\end{gathered}
$$

with either $u=1$ (subtype 2.N.1), $u=0$ and $w=1$ (subtype 2.N.O1), or $u=w=0$ (subtype 2.N.OO).

As $\Upsilon^{1}=\Upsilon^{2}$, the collineation equations (27) imply that $\mathcal{L}_{\mathbf{X}} \Upsilon^{1}=b \Upsilon^{1}$. Besides, their integrability conditions are

$$
\mathcal{L}_{\mathbf{X}} d \phi^{\alpha}=D_{\beta}^{\alpha}\left(b d \phi^{\beta}+d b \wedge \phi^{\beta}\right)
$$

and it can be easily proven that

Proposition 4: The necessary condition for Eqs. (27) and (45) to have a solution is

(0): if $u=0$, and therefore $\phi^{2}-\phi^{1}$ is integrable, then

$$
d b=w \mathcal{L}_{\mathbf{X}} \phi^{3}+\tilde{b}_{\alpha} \phi^{\alpha}, \quad w=0, \quad \text { or } \quad 1
$$

(1): if $u=1$, then

$$
\begin{gathered}
\mathcal{L}_{\mathbf{X}} \phi^{3}=-2 b \phi^{3}, \\
\mathcal{L}_{\mathbf{X}}\left(\phi^{4}-w \phi^{2}\right)=3 b\left(\phi^{4}-w \phi^{2}\right)-2 b \phi^{2}+f \phi^{3}, \\
d b=-b(1+2 w) \phi^{3}+\frac{1}{2}(\mathbf{X} t-b s) \phi^{1}-\frac{1}{2}(\mathbf{X} s-b t) \phi^{2},
\end{gathered}
$$

where $f$ is some function.

Subtype 2.N.1: This corresponds to $u=1$, then in the base

$$
\hat{\phi}^{i}:=\phi^{i}, \quad i=1,2,3, \quad \text { and } \quad \hat{\phi}^{4}:=\phi^{4}-w \phi^{2},
$$

Eqs. (27), and (47)-(49) read

$$
\left.\begin{array}{l}
\mathcal{L}_{\mathbf{X}} \hat{\phi}^{a}=\left(b U_{c}^{a}+f \delta_{4}^{a} \delta_{c}^{3}\right) \hat{\phi}^{c} \\
d b=\frac{1}{2}\left(\mathbf{X} B_{c}+b E_{c}\right) \hat{\phi}^{c}
\end{array}\right\},
$$

where all $U_{c}^{a}$ vanish, except $U_{2}^{1}=U_{1}^{2}=1, U_{3}^{3}=U_{2}^{4}=-2$, and $U_{4}^{4}=3$, and

$$
B_{c}=(t,-s, 2 w, 0), \quad E_{c}=(-s, t,-2,-4 w) .
$$

Let $\left\{\mathbf{Y}_{a}\right\}$ be the dual base of $\left\{\hat{\phi}^{a}\right\}$, and write $d \hat{\phi}^{a}=-\frac{1}{2} \hat{C}_{c b}^{a} \hat{\phi}^{c} \wedge \hat{\phi}^{b}$, and $\mathbf{X}=\hat{X}^{a} \mathbf{Y}_{a}$. Equation (50) is then a partial differential system on the unknowns $\hat{X}^{a}$ and $b$ which, due to the presence of $f$, is not in closed form, but the integrability conditions may help to determine it. Indeed, by a similar technique as for case 2.I.b.1 in Subsection V B, we can easily conclude that, whenever 
$\hat{\phi}^{3} \wedge d \hat{\phi}^{3} \neq 0$ or $\hat{\phi}^{3} \wedge d\left(\hat{C}_{4 \beta \hat{\phi}^{\beta}}^{4}\right) \neq 0, \mathcal{C}_{T}$ is a Lie algebra and $\operatorname{dim} \mathcal{C}_{T} \leq 5$. Like then, we do not consider the residual nongeneric subcase when $f$ cannot be derived from the integrability conditions of Eq. (50).

For subtypes 2.N.01 and 2.N.00, we have to take into account that

Proposition 5: If $u=0$ and $s+t=2 r$, a T-frame can be found such that $\tilde{t}=\tilde{s}=\tilde{r}$.

Proof: If $u=0$, by Eqs. (42) and (44), the condition $t+s-2 r=0$ is $T$-rotation invariant and, using the transformation (24), we easily arrive at

$$
(\tilde{t}-\tilde{s}) e^{\zeta} \Upsilon^{1}=(t-s) \Upsilon^{1}+2 d \zeta \wedge\left(\phi^{1} \wedge d \phi^{2}-\phi^{2} \wedge d \phi^{1}\right)
$$

and suitably choosing $\zeta$ we can make $\tilde{t}=\tilde{s}$.

Subtype 2.N.01: This corresponds to $u=0, w=1$ and, including Proposition 5, Eq. (44) reads

$$
d\left(\phi^{2}-\phi^{1}\right)=\left(\phi^{2}-\phi^{1}\right) \wedge \phi^{3} .
$$

After a little algebra, the integrability conditions for Eq. (27) then yield:

$$
\mathcal{L}_{\mathbf{X}} \hat{\phi}^{a}=U_{b}^{a} \hat{\phi}^{b}+f \delta_{4}^{a} \hat{\phi}^{3},
$$

where all $U_{b}^{a}$ vanish except

$$
U_{2}^{1}=U_{1}^{2}=b, \quad U_{3}^{3}=b_{3}, \quad U_{4}^{3}=b_{4}, \quad U_{4}^{4}=b-b_{3},
$$

$b$ is a solution of the partial differential equation $b_{1}+b_{2}+b_{4}=0$ and $\hat{\phi}^{a}$ are defined as above.

Choosing any $b$ fulfilling the latter, we have to solve the partial differential system (51), which is not in closed form due to the arbitrary function $f$. To discuss its solution, we should proceed as for the PDS (50) in subtype 2.N.1.

Subtype 2.N.00: This corresponds to $u=w=0$ and, including Proposition 5, Eqs. (43) and (44) read

$$
d \phi^{1}=\frac{t}{2} \phi^{1} \wedge \phi^{2}+\phi^{3} \wedge \phi^{4}, \quad d\left(\phi^{2}-\phi^{1}\right)=0 .
$$

Therefore, a function $y$ exists such that $\phi^{2}-\phi^{1}=d y$ and Eq. (27) implies that

$$
\mathbf{X} y=B(y), \quad b=-B^{\prime}(y),
$$

where $B(y)$ is some one variable function and $B^{\prime}$ its derivative.

Case 2.N.00.nd: If $t \neq 0$, then $\Sigma^{11} \neq 0, d \phi^{1}$ is symplectic and canonical coordinates exist such that $\phi^{1}=p_{i} d q^{i}$. Writing the collineation equation (27) in these coordinates, we easily arrive at

$$
\mathbf{X}=-\left(B^{\prime} p_{i}+\partial_{i} F\right) \partial^{i}+\partial^{i} F \partial_{i}=-\{F,-\}-B^{\prime} p_{i} \partial^{i},
$$

where $F$ is a solution of $p_{j} \partial^{j} F-F=B(y)$, that is,

$$
F=z F_{0}\left(q^{i}, p_{2} / p_{1}\right)+z \int \frac{B(y)}{z^{2}} d z
$$

where the variable $z^{2}:=p_{1}^{2}+p_{2}^{2}$ has been introduced.

Case 2.N.OO.d: If $t=0$, then $d \phi^{1} \wedge d \phi^{1}=0$ but $d \phi^{1} \wedge \phi^{1} \neq 0$ and canonical coordinates can be chosen such that

$$
\phi^{1}=d q^{2}+p_{1} d q^{1} \quad \text { and } \quad \phi^{2}-\phi^{1}=d p_{2} .
$$

Writing the collineation equation (27) in these coordinates, we easily obtain that

$$
\mathbf{X}=\left(b p_{1}-\partial_{1} F_{0}\right) \partial^{1}+F \partial_{2}+X_{2} \partial^{2},
$$


where $b$ is a constant, $X_{2}$ is arbitrary and

$$
F=b\left(q^{2}+p_{2}\right)+F_{0}\left(q^{j}\right)
$$

\section{Type 2.H}

In this case, $\Upsilon^{\alpha}=0, \alpha=1,2, T$ is holonomous and coordinates $x^{a}, a=1 \ldots 4$, exist such that

$$
T=T_{\alpha \beta}\left(x^{a}\right) d x^{\alpha} \otimes d x^{\beta}, \quad \text { with } \quad \operatorname{det} T_{\alpha \beta} \neq 0,
$$

and three cases arise depending on

$$
m:=\operatorname{rank}\left\{d x^{1}, d x^{2}, d T_{\alpha \beta}\right\}, \quad 2 \leq m \leq 4 .
$$

Case 2.H.0: If $m=2$, then $\partial_{A} T_{\alpha \beta}=0, A=3,4$.

Case 2.H.1: If $m=3$, the coordinates can be chosen so that $T_{11}=x^{3}, \quad T_{12}=u\left(x^{3}\right), \quad T_{22}=$ $v\left(x^{3}\right)$.

Case 2.H.2: If $m=4$, the coordinates can be chosen so that $T_{11}=x^{3}, \quad T_{12}=u, \quad T_{22}=x^{4}$.

Type $\mathbf{2 . H}$ tensors will be dealt in much the same way as rank 3 tensors. We first write the collineation field as $\mathbf{X}=\mathbf{Z}+f^{A} \partial_{A}$, where $f^{A}$ are two functions and $\mathbf{Z}=Z^{\alpha} \partial_{\alpha}$ is tangential to the submanifolds $x^{B}=$ constant, $A, B=3,4$.

It is obvious that $T_{A b}=0$, which, including Eq. (1), implies that

$$
\partial_{A} Z^{\alpha}=0, \quad \nabla_{\alpha} Z_{\beta}=\Omega_{\alpha \beta}-f^{A} K_{A \mid \alpha \beta}=0,
$$

where $\Omega_{(\alpha \beta)}=0, K_{A \mid \alpha \beta}:=\frac{1}{2} \partial_{A} T_{\alpha \beta}$, and $\nabla$ is the Levi-Civita connection for the 2-metric $T_{\alpha \beta}$ on the surfaces $x^{B}=$ constant. The second of these equations looks like a non-homogeneous Killing equation (parametrized with $x^{B}$ ) and the question is: does it admit solutions $Z^{\alpha}$ that do not depend on $x^{B}$ for some appropriate $f^{A}$ ?

In case 2.H.0, $K_{A \mid \alpha \beta}=0, A=3,4$, the answer is obvious because coordinates $x^{3}$ and $x^{4}$ are mere parameters and Eq. (57) reduces to a Killing equation in 2 dimensions. The collineation field is then $\mathbf{X}=\mathbf{Z}+f^{A} \partial_{A}$, where $f^{A}$ are arbitrary and $\mathbf{Z}$ is a Killing vector for the non-degenerate rank 2 metric $T$ in each submanifold $x^{B}=$ constant.

In case 2.H.1, $K_{3 \mid \alpha \beta} \neq 0$ and $K_{4 \mid \alpha \beta}=0$. Then Eq. (57) does not involve the function $f^{4}$, which is arbitrary. The coordinate $x^{4}$ is only a parameter and the problem has reduced to finding the collineation fields of a rank two tensor on each submanifold $x^{4}=$ constant, which is similar to the problem treated in Sec. III.

The generic case is 2.H.2, i.e., $K_{A \mid \alpha \beta} \neq 0, A=3,4$,

$$
K_{3 \mid \alpha \beta}=\frac{1}{2}\left(\begin{array}{cc}
1 & u_{\mid 3} \\
u_{\mid 3} & 0
\end{array}\right), \quad K_{4 \mid \alpha \beta}=\frac{1}{2}\left(\begin{array}{cc}
0 & u_{\mid 4} \\
u_{\mid 4} & 1
\end{array}\right),
$$

where $u_{\mid A}:=\partial_{A} u$.

Similarly as in Sec. III, the integrability conditions for Eq. (57) following from the commutation relations for $\nabla_{\alpha}$ and $\nabla_{\mu}$ imply that

$$
\nabla_{\mu} \Omega_{\kappa \lambda}=R Z_{[\lambda} T_{\kappa] \mu}+2 \nabla_{[\lambda}\left(f^{A} K_{A \mid \kappa] \mu}\right)
$$

and also an infinite hierarchy of conditions on $Z^{\alpha}, \Omega_{\alpha \beta}$, and $f$.

As for the commutation of the derivatives $\partial_{A}$ and $\nabla_{\alpha}$ applied to $Z_{\alpha}$, we readily obtain that

$$
\begin{aligned}
& \left.\partial_{A} \Omega_{\alpha \beta}=2 \Omega_{\lambda[\beta} K_{A \mid \alpha]}^{\lambda}+2 Z^{\nu} \nabla_{[\alpha} K_{A \mid \beta] \nu}-2 f^{B} K_{A \mid \nu[\alpha} K_{B \mid} \stackrel{\nu}{\beta}\right], \\
& \left.\partial_{A} f^{B} K_{B \mid \alpha \beta}+f^{B}\left(\partial_{A} K_{B \mid \alpha \beta}-2 K_{A \mid v(\alpha} K_{B \mid} \stackrel{\nu}{\beta}\right)\right)+Z^{\nu} \nabla_{\nu} K_{A \mid \alpha \beta}+2 K_{A \mid v(\alpha} \Omega_{\beta)}^{\nu}=0 .
\end{aligned}
$$

In the generic case, the latter can be solved in $f^{B}=F^{B}(Z, \Omega)$ and in $\partial_{A} f^{B}=F_{A}^{B}(Z, \Omega)$, which permits to close the system (57) to (59), whence it follows that $\mathcal{C}_{T}$ is a Lie algebra and $\operatorname{dim} \mathcal{C}_{T} \leq 3$.

The non-generic case requires further study which we shall not tackle here. 


\section{APPLICATION}

As an application, we study the Ricci collineations of $2+2$ type B warped spacetimes

$$
d s^{2}=g_{A B}\left(x^{C}\right) d x^{A} d x^{B}+h^{2}\left(x^{C}\right) g_{i j}\left(x^{k}\right) d x^{i} d x^{j}
$$

with $A, B=3,4, i, j \ldots=1,2$.

The non-vanishing components of the Ricci tensor are

$$
R_{A B}=\frac{1}{2} R_{1} g_{A B}-\frac{2}{h} D_{A} h_{B}, \quad R_{i j}=F g_{i j},
$$

where $F:=\frac{1}{2}\left(R_{2}-D^{A} D_{A} h^{2}\right)$, and $R_{1}\left(x^{B}\right)$ and $R_{2}\left(x^{k}\right)$ respectively are the Ricci scalars for the 2-dimensional metrics $g_{A B}$ and $g_{i j}$.

If $F \cdot \operatorname{det}\left(R_{A B}\right) \neq 0$, then the Ricci tensor has rank 4 and the Ricci collineations conform a Lie algebra whose dimension is at most ten. This case has been completely solved in Ref. 11 and we shall confine ourselves to the case when the rank is less than 4 , which will serve as a test for the power of our approach.

\section{A. The case $R_{A B}=0$}

Apart from trivial case $F=0$ (and Ric $=0$ ), we have that, due to the low number of dimensions, coordinates can be chosen so that

$$
\text { Ric }= \pm e^{f} \eta_{i j} d x^{i} \otimes d x^{j}, \quad \eta_{i j}=\operatorname{diag}(1, \sigma)
$$

with $\sigma= \pm 1$ and $f:=\log |F|$.

The Ricci collineation condition then implies that

$$
\partial_{i} X_{j}+\partial_{j} X_{i}+\mathbf{X} f \eta_{i j}=0, \quad \partial_{B} X_{i}=0
$$

with $X_{i}:=\eta_{i j} X^{j}$, whence it follows that

$$
\mathbf{X}=\partial_{1} U \partial_{1}-\partial_{2} U \partial_{2}+X^{B} \partial_{B},
$$

where $U\left(x^{b}\right)$ is a solution of $\eta^{i j} \partial_{i} \partial_{j} U=0$ and

$$
\mathbf{X} f=-\partial_{1}^{2} U+\sigma \partial_{2}^{2} U .
$$

In case that $\partial_{A} f \neq 0$, the latter is a constraint connecting the two components $X^{B}$, whereas if $\partial_{A} f=$ 0 , it yields a further constraint on $U\left(x^{b}\right)$.

\section{B. The case $F \neq 0$ and $\operatorname{rank} R_{A B}=1$}

The Ricci tensor has rank 3 and coordinates exist such that

$$
\text { Ric }=H\left(x^{C}\right) d x^{3} \otimes d x^{3} \pm e^{f} \eta_{i j} d x^{i} \otimes d x^{j}
$$

and the results obtained in Sec. III apply, with the non-vanishing components of $T_{\alpha \beta}$ and $K_{\alpha \beta}$ given by

$$
\begin{aligned}
& T_{i j}= \pm e^{f} \eta_{i j}, \quad T_{33}=H, \\
& 2 K_{i j}= \pm \partial_{4} f e^{f} \eta_{i j}, \quad 2 K_{33}=\partial_{4} H,
\end{aligned}
$$

(a) If $\partial_{4} H=\partial_{4} f=0$, then it belongs to the subtype $\mathbf{3 . 0}$ and the class of Ricci collineations has an infinite number of dimensions.

However, due to the simplicity of this particular case we can go further, $x^{3}$ can be chosen so that $H= \pm 1$ and Eq. (7) reads

$$
\left.\begin{array}{l}
e^{f} \partial_{3} Z_{i}+\partial_{i} Z^{3}=0, \quad \partial_{3} Z^{3}=0 \\
\partial_{i} Z_{j}+\partial_{j} Z_{i}+\mathbf{Z} f \eta_{i j}=0
\end{array}\right\}
$$


with $Z_{i}:=\eta_{i j} Z^{i}$. As before, the solution of the third equation is

$$
Z_{1}=\partial_{1} U, \quad Z_{2}=-\partial_{2} U, \quad \eta^{i j} \partial_{i} \partial_{j} U=0
$$

with

$$
Z^{3} \partial_{3} f+\partial_{1} U \partial_{1} f-\partial_{2} U \partial_{2} f+\partial_{1}^{2} U-\sigma \partial_{2}^{2} U=0 .
$$

If $\partial_{3} f \neq 0$, this determines $Z^{3}$ and the other two equations (62) are constraints on $f$ and $U$.

If, on the contrary $\partial_{3} f=0$, the consistency of the system (62) leads to

$$
U=x^{3} A\left(x^{j}\right)+B\left(x^{j}\right)+V\left(x^{3}\right),
$$

where $A$ and $B$ are two solutions of

$$
f_{1} \partial_{1} M-f_{2} \partial_{2} M+2 \partial_{11} M=0, \quad \eta^{i j} \partial_{i} \partial_{j} M=0 .
$$

(b) If $\partial_{4} f \neq 0$ or $\partial_{4} H \neq 0$, then $K_{\alpha \beta} \neq 0$. If besides $\partial_{4}^{2} F \partial_{4} H \neq \partial_{4}^{2} H \partial_{4} F$, then $\dot{K}_{\alpha \beta}$ is not proportional to $K_{\alpha \beta}$ and Eq. (13) can be solved for $f$, we are in the subtype $\mathbf{3 . 1}$ and the class $\mathcal{C}_{T}$ of collineation fields is a Lie algebra whose dimension is at most 6.

Otherwise, if $\partial_{4}^{2} F \partial_{4} H=\partial_{4}^{2} H \partial_{4} F$, the linear system (15) does not determine $f_{\mid i}$, Ric belongs to the non-generic case left unsolved in Sec. $\mathrm{V}$ and the collineation field might include arbitrary functions.

\section{The case $\boldsymbol{F}=0$}

If the rank of $R_{A B}$ is 1 , we have that Ric $=\phi \otimes \phi$, where $\phi=\phi_{A}\left(x^{B}\right) d x^{A}$ is integrable due to the low number of dimensions, coordinates exist such that $\phi=p d q$ and we are in the type 1.d.h.

If the rank of $R_{A B}$ is 2 , we are in the type $\mathbf{2 . H}$, with Ric $=R_{A B}\left(x^{C}\right) d x^{A} \otimes d x^{B}$. Writing the collineation field as $\mathbf{X}=\mathbf{Z}+f^{i} \partial_{i}$, we have that $\mathbf{Z}=Z^{A}\left(x^{B}\right) \partial_{B}$ is a Killing vector of $R_{A B}$ taken as a 2-dimensional non-degenerate metric and $f$ are arbitrary functions.

\section{ACKNOWLEDGMENTS}

The author is indebted to J. Carot for calling his attention on the subject and for stimulating comments and discussions. Also the author is grateful for the warm hospitality of the Edinburgh Mathematical Physics Group (Edinburgh University), where a significant part of the present work was developed during a sabbatical stay. This work is supported by Ministerio de Educacion y Ciencia through Grant No. FIS2007-63034 and by Generalitat de Catalunya, 2009SGR-417 (DURSI).

${ }^{1}$ H. Stefani, D. Kramer, M. MacCallum, C. Hoenselaers, and E. Herlt, Exact Solutions of Einstein's Field Equations (Cambridge University Press, Cambridge, 2003).

${ }^{2} \mathrm{~K}$. Yano, The Theory of Lie Derivatives and Its Applications (North-Holland, Amsterdam, 1955).

${ }^{3}$ G. S. Hall, J. Math. Phys. 31, 1198 (1990).

${ }^{4}$ G. S. Hall and D. P. Lonie, Class. Quantum Grav. 12, 1007 (1995).

${ }^{5}$ A. H. Bokhari and A. Qadir, J. Math. Phys. 34, 3543 (1993).

${ }^{6}$ A. Melfo, L. Nuñez, U. Percoco, and V. M. Villalba, J. Math. Phys. 33, 2258 (1992).

${ }^{7}$ G. S. Hall and J. da Costa, J. Math. Phys. 32, 2848 (1991); 32, 2854 (1991).

${ }^{8}$ J. Carot, J. da Costa, and E. G. L. R. Vaz, J. Math. Phys. 35, 4832 (1994).

${ }^{9}$ G. H. Katzin, J. Levine, and W. R. Davis, J. Math. Phys. 10, 617 (1969).

${ }^{10}$ C. Godbillon, Géometrie Différentielle et Mécanique analytique (Hermann, Paris, 1969).

${ }^{11}$ J. L. Flores, Y. Parra, and U. Percoco, J. Math. Phys. 45, 3546 (2004). 